\title{
Modafinil improves monocrotaline-induced pulmonary hypertension rat model
}

\author{
Hyeryon Lee', Kwan Chang Kim², Min-Sun Cho ${ }^{3}$, Suk-hyo Suh ${ }^{4}$ and Young Mi Hong
}

\begin{abstract}
BACKGROUND: Pulmonary arterial hypertension (PAH) progressively leads to increases in pulmonary vasoconstriction. Modafinil plays a role in vasorelaxation and blocking KCa3.1 channel with a result of elevating intracellular cyclic adenosine monophosphate (cAMP) levels. The purpose of this study is to evaluate the effects on modafinil in monocrotaline (MCT)-induced PAH rat.
\end{abstract}

METHODS: The rats were separated into three groups: the control group, the monocrotaline (M) group (MCT $60 \mathrm{mg} / \mathrm{kg}$ ), and the modafinil (MD) group (MCT $60 \mathrm{mg} / \mathrm{kg}+$ modafinil).

RESULTS: Reduced right ventricular pressure (RVP) was observed in the MD group. Right ventricular hypertrophy was improved in the MD group. Reduced number of intra-acinar pulmonary arteries and medial wall thickness were noted in the MD group. After the administration of modafinil, protein expressions of endothelin-1 (ET-1), endothelin receptor A (ERA) and KCa3.1 channel were significantly reduced. Modafinil suppressed pulmonary artery smooth muscle cell (PASMC) proliferation via CAMP and KCa3.1 channel. Additionally, we confirmed protein expressions such as Bcl-2-associated $\mathrm{X}$, vascular endothelial growth factor, tumor necrosis factor- $\alpha$, and interleukin-6 were reduced in the MD group.

CONCLUSION: Modafinil improved PAH by vasorelaxation and a decrease in medial thickening via ET-1, ERA, and KCa3.1 down regulation. This is a meaningful study of a modafinil in PAH model.

$\mathbf{P}$ ulmonary arterial hypertension (PAH) is a refractory disease and all occurrences of PAH exhibit increased pulmonary vascular resistance and pulmonary vascular remodeling $(1,2)$. The disease is a progressive condition characterized by elevated pulmonary arterial pressures leading to right ventricular failure (3). Although the pathogenesis of PAH is not fully understood, the histopathological characteristics include intimal lesions, medial hypertrophy, and adventitial thickening of precapillary pulmonary arteries (2). In PAH patients, different degrees of medial hypertrophy were noted in small pulmonary arteries, ultimately leading to the obliteration of the vessels (2). Hyperplasia of pulmonary artery smooth muscle cells (PASMCs) is the main element of these changes $(4,5)$. Also, there is evidence that the intermediate-conductance calciumactivated potassium channel (KCa3.1 channel) is associated with smooth muscle proliferation (6-8). However, it is still a controversial question whether there is a correlation between KCa3.1 channel and PAH.

Many experimental animal models of PAH exist and this includes monocrotaline (MCT)-induced and chronic hypoxiainduced rats (9-11). Although the precise mechanism by which MCT leads to PAH is unknown, the MCT-induced PAH model is a mainstay in evaluating experiments of new therapeutic interventions. MCT models can be created by a single subcutaneous or intraperitoneal injection in rats (12). MCTinduced PAH models shows excessive proliferation of pulmonary arterial smooth muscle cells.

Current therapeutic strategies for decreasing $\mathrm{PAH}$ include calcium channel blockers, endothelin (ET) receptor antagonists, phosphodiesterease type 5 inhibitors, and prostanoid. Calcium channel blocker induces vasodilation through the decrease in intracellular calcium concentration (1). ET receptor antagonist has vasodilation and anti-proliferative properties but it is expensive. Phosphodiesterease type 5 inhibitor also induces vasodilation and antiproliferative properties by inhibiting cyclic guanosine monophosphate degradation. However, no data is currently available on the long-term efficacy of the lower dosage. Prostanoid is a cyclic adenosine monophosphate (cAMP)-dependent agent that induces vasodilation and an antiproliferative property. It is difficult for oral administration, it has a short half-life and it is expensive. These current therapies have an effect on PAH but the prognosis of PAH remains poor (13-16). The current medications have limitations because they provide only symptomatic alleviation. Survival rate continues to be poor despite treatment, so developing a new drug for $\mathrm{PAH}$ is urgent (17).

Modafinil (Provigil; Cephalon, West Chester, PA) is an agent that has been approved by the Food and Drug Administration for narcolepsy $(18,19)$. It has been described that modafinil has an effect on cAMP-induced decrease in KCa3.1 currents or in vascular smooth muscle contraction (20). In comparison with iloprost, the advantages of modafinil are that it is available for oral administration, it has a long half-life and it

\footnotetext{
'Department of Pediatrics, Ewha Womans University School of Medicine, Seoul, Republic of Korea; ${ }^{2}$ Department of Thoracic and Cardiovascular Surgery, Ewha Womans University School of Medicine, Seoul, Republic of Korea; ${ }^{3}$ Department of Pathology, Ewha Womans University School of Medicine, Seoul, Republic of Korea; ${ }^{4}$ Department of Physiology, Ewha Womans University School of Medicine, Seoul, Republic of Korea. Correspondence: Young Mi Hong (ymhong@ewha.ac.kr)

Received 18 June 2015; accepted 16 December 2015; advance online publication 27 April 2016. doi:10.1038/pr.2016.38
} 
is inexpensiveness. However, there has been no report about modafinil in PAH.

It has been reported that cell proliferation is regulated by KCa3.1 channel in SMCs. There are several studies about the effect of KCa3.1 channels blocker on human lung mast cell proliferation and migration $(21,22)$. However, there have not been any reports about the effect of modafinil on PASMCs proliferation.

The purposes of this study were to determine the optimal dosage of modafinil to improve $\mathrm{PAH}$ and to investigate the effect of modafinil on right ventricle pressure (RVP), right ventricular hypertrophy, pathology, and protein expressions in MCT-induced $\mathrm{PAH}$ rat.

\section{RESULTS}

\section{RVP Was Reduced After Modafinil Treatment}

RVP was increased in the $M$ group compared to the $C$ group at weeks 2 (C vs. $\mathrm{M} ; 10.67 \pm 0.58$ vs. $27.33 \pm 3.79 \mathrm{mmHg}, P<0.05$ ) and 4 (C vs. $\mathrm{M} ; 10.67 \pm 1.53$ vs. $31.75 \pm 2.50 \mathrm{mmHg}, P<0.05)$.

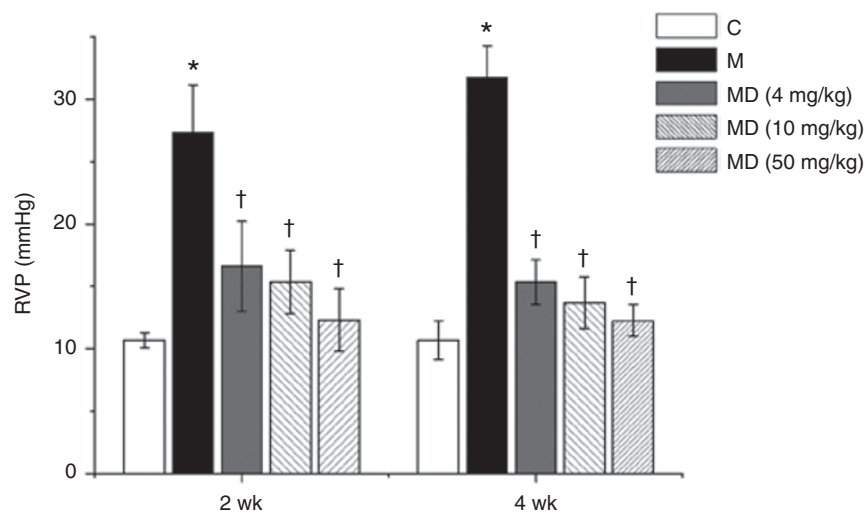

Figure 1. Changes in RVP after modafinil administration in MCT-induced PAH rat. RVP was decreased in all of the MD groups at weeks 2 and 4 . There was no significant difference among the MD groups. $C$, control group; $M$, monocrotaline group; $\mathrm{MCT}$, monocrotaline; $\mathrm{MD}$, modafinil group; $\mathrm{PAH}$, pulmonary arterial hypertension; RVP, right ventricular pressure. ${ }^{*} P<0.05$ C vs. $M,{ }^{\dagger} P<0.05, M$ vs. MD.
We compared changes of RVP according to the size of the dose of modafinil. As administrations of modafinil, RVP was significantly reduced at week 2 (M vs. MD $(4 \mathrm{mg} / \mathrm{kg})$ vs. $\mathrm{MD}(10 \mathrm{mg} / \mathrm{kg})$ vs. $\mathrm{MD}(50 \mathrm{mg} / \mathrm{kg}) ; 27.33 \pm 3.79 \mathrm{mmHg}$ vs. $16.60 \pm 3.61 \mathrm{mmHg}$ vs. $15.33 \pm 2.52 \mathrm{mmHg}$ vs. $12.33 \pm 2.52$ $\mathrm{mmHg}, P<0.05)$ and $4(\mathrm{M}$ vs. $\mathrm{MD}(4 \mathrm{mg} / \mathrm{kg})$ vs. $\mathrm{MD}(10 \mathrm{mg} /$ $\mathrm{kg})$ vs. $\mathrm{MD}(50 \mathrm{mg} / \mathrm{kg}) ; 31.75 \pm 2.50 \mathrm{mmHg}$ vs. $15.33 \pm 1.80$ $\mathrm{mmHg}$, vs. $13.67 \pm 2.08 \mathrm{mmHg}$ vs. $12.25 \pm 1.26 \mathrm{mmHg}$, $P<0.05)$ (Figure 1). Modafinil had an effect on RVP, but there were no significant changes according to the dose of modafinil. These data suggest that modafinil treatment has a beneficial effect on PAH.

\section{RV Hypertrophy Was Decreased After Modafinil Treatment}

$\mathrm{RV} /$ body weight $(\mathrm{BW})$ was also increased in the $\mathrm{M}$ group at weeks 2 and $4(P<0.05)$ compared with the $C$ group (Table 1$)$.

$\mathrm{RV} / \mathrm{BW}$ significantly reduced in the MD group compared with the $\mathrm{M}$ group at week 4 (M vs. $\mathrm{MD}(4 \mathrm{mg} / \mathrm{kg})$ vs. $\mathrm{MD}$ $(10 \mathrm{mg} / \mathrm{kg})$ vs. $\mathrm{MD}(50 \mathrm{mg} / \mathrm{kg}) ; 1.76 \pm 0.14$ vs. $1.45 \pm 0.14$, vs. $1.30 \pm 0.30$ vs. $1.28 \pm 0.26, P<0.05)$.

$\mathrm{RV} / \mathrm{LV}+\mathrm{S}$ ratio was lager in the $\mathrm{M}$ group compared with the $\mathrm{C}$ group at weeks 2 and $4(P<0.05)$. RV/LV $+S$ ratio was reduced in the MD group compared to the $\mathrm{M}$ group at week 4 (M vs. $\mathrm{MD}(4 \mathrm{mg} / \mathrm{kg})$ vs. MD $(10 \mathrm{mg} / \mathrm{kg})$ vs. MD $(50 \mathrm{mg} / \mathrm{kg}) ; 0.90 \pm 0.17$ vs. $0.72 \pm 0.14$ vs. $0.67 \pm 0.11$ vs. $0.52 \pm 0.15, P<0.05)$. Thus, the results suggest that modafinil attenuates $\mathrm{RV}$ hypertrophy due to the decrease in RVP in MCT-induced PAH rats as shown in Figure 2 .

\section{Changes of Pathology According to the Dose of Modafinil in MCT-Induced PAH Rat}

In terms of pulmonary pathology, medial wall thickness of the pulmonary arterioles was increased in the $\mathrm{M}$ group compared to the $\mathrm{C}$ group at weeks 2 and 4 . Because it was a first trial on the effects of modafinil on $\mathrm{PAH}$, we had to consider what dose to test. We decided to try: 4,10 , and $50 \mathrm{mg} / \mathrm{kg}$. We demonstrated that at $4 \mathrm{mg} / \mathrm{kg}$ of modafinil, small pulmonary artery's medial wall thickness was reduced at week 2 (M vs. MD $(4 \mathrm{mg} / \mathrm{kg}$ ) vs.

Table 1. Changes of body and organ weight after modafinil administration in MCT-induced PAH rat

\begin{tabular}{|c|c|c|c|c|c|}
\hline Week & Group & $\mathrm{BW}(\mathrm{g})$ & $\mathrm{RV} / \mathrm{BW}$ & LV/BW & Lung/BW \\
\hline \multirow[t]{4}{*}{2} & C & $357.50 \pm 11.65$ & $0.58 \pm 0.02$ & $2.16 \pm 0.05$ & $4.20 \pm 0.27$ \\
\hline & M & $291.50 \pm 15.39^{*}$ & $0.81 \pm 0.08^{*}$ & $2.24 \pm 0.18$ & $11.21 \pm 5.10^{*}$ \\
\hline & $\mathrm{MD}(10 \mathrm{mg} / \mathrm{kg})$ & ${ }^{5} 307.83 \pm 19.19$ & $0.91 \pm 0.03$ & $2.21 \pm 0.03$ & $14.28 \pm 6.51$ \\
\hline & $\mathrm{MD}(50 \mathrm{mg} / \mathrm{kg})$ & $263.00 \pm 29.48^{\dagger}$ & $0.84 \pm 0.17$ & $2.21 \pm 0.13$ & $9.18 \pm 6.06$ \\
\hline \multirow{4}{*}{4} & M & $356.25 \pm 30.42^{*}$ & $1.76 \pm 0.14^{*}$ & $1.97 \pm 0.14$ & $8.89 \pm 6.18$ \\
\hline & $\mathrm{MD}(4 \mathrm{mg} / \mathrm{kg})$ & $332.75 \pm 10.26$ & $1.45 \pm 0.14^{\dagger}$ & $2.20 \pm 0.20$ & $14.71 \pm 6.97$ \\
\hline & $\mathrm{MD}(10 \mathrm{mg} / \mathrm{kg})$ & $344.17 \pm 27.88$ & $1.30 \pm 0.30^{\dagger}$ & $2.30 \pm 0.11$ & $9.25 \pm 0.50$ \\
\hline & $\mathrm{MD}(50 \mathrm{mg} / \mathrm{kg})$ & $322.38 \pm 17.41$ & $1.28 \pm 0.26^{\dagger}$ & $2.48 \pm 0.30$ & $8.41 \pm 3.48$ \\
\hline
\end{tabular}

Data are given as mean \pm SD.

$B W$, body weight; $C$, control; LV, left ventricle; $M$, monocrotaline; $M D$, modafinil; $R V$, right ventricle.

${ }^{*} P<0.05$ C vs. $M,{ }^{\dagger} P<0.05 \mathrm{M}$ vs. MD, ${ }^{\S} P<0.05$ among the MD groups. 
$\mathrm{MD}(10 \mathrm{mg} / \mathrm{kg}) ; 42.33 \pm 0.79$ vs. $30.95 \pm 0.06$ vs. $36.64 \pm 0.05$, $P<0.05)$ and $4(\mathrm{M}$ vs. $\mathrm{MD}(4 \mathrm{mg} / \mathrm{kg})$ vs. $\mathrm{MD}(10 \mathrm{mg} / \mathrm{kg})$ vs. $\mathrm{MD}(50 \mathrm{mg} / \mathrm{kg}) ; 42.35 \pm 1.94$ vs. $34.78 \pm 0.01$ vs. $31.39 \pm 0.37$ vs. $36.32 \pm 1.00, P<0.05)$. Result from neovascularization, the number of intra acinar arteries was increased at weeks 2 and $4(P<0.05)$. In terms of the effect of modafinil treatment, the number of intra acinar arteries was reduced in the MD group at weeks 2 (M vs. MD (10 mg/kg) vs. MD (50 mg/kg);

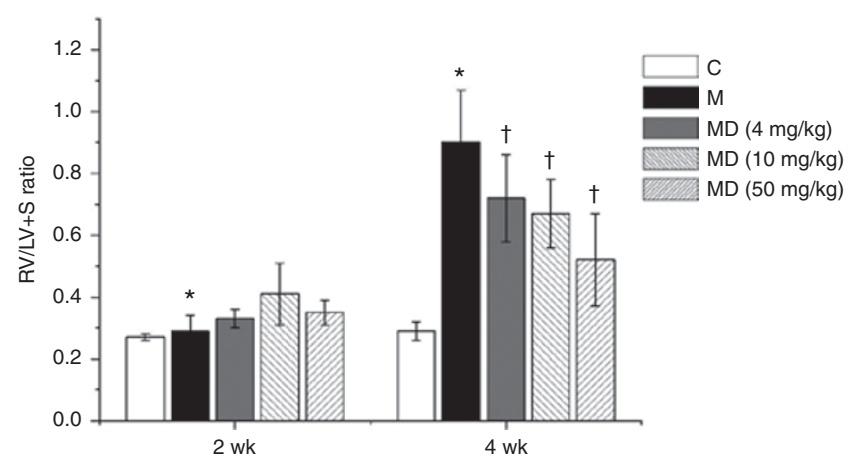

Figure 2. Changes of RV hypertrophy after modafinil administration in $\mathrm{MCT}$-induced $\mathrm{PAH}$ rat. RV/LV+S ratio was significantly decreased in the all of the MD groups at week 4. C, control group; $M$, monocrotaline group; $M C T$, monocrotaline; $M D$, modafinil group; $\mathrm{PAH}$, pulmonary arterial hypertension; RV, right ventricle. ${ }^{*} P<0.05 \mathrm{C}$ vs. $M,{ }^{+} P<0.05 \mathrm{M}$ vs. MD.
$1.70 \pm 0.15$ vs. $1.39 \pm 0.46$ vs. $1.13 \pm 0.05$ vs. $1.41 \pm 0.05, P<0.05)$ and $4(\mathrm{M}$ vs. $\mathrm{MD}(4 \mathrm{mg} / \mathrm{kg})$ vs. $\mathrm{MD}(10 \mathrm{mg} / \mathrm{kg})$ vs. MD (50 mg/ $\mathrm{kg}) ; 2.40 \pm 0.10$ vs. $1.22 \pm 0.06$ vs. $1.20 \pm 0.27$ vs. $1.82 \pm 0.27$, $P<0.05$ )(Figure 3). Present data suggests modafinil positively affects pulmonary vascular remodeling by inhibiting excessive proliferation of the medial wall and as we mentioned earlier it improved pulmonary hemodynamics. Overall, MD $(50 \mathrm{mg}$ / $\mathrm{kg}$ ) had no effect in the reduction of medial wall thickening at week 2 and MD $(4 \mathrm{mg} / \mathrm{kg})$ had no effect in the reduction of neovascularization at week 2 as well. Therefore, we considered that MD $(10 \mathrm{mg} / \mathrm{kg})$ is the most appropriate dose. So, we proceeded with the second experiments using MD $(10 \mathrm{mg} / \mathrm{kg})$ group tissues.

\section{The Survival Rate Improved After Modafinil Treatment}

The survival rate reduced in the $M$ group compared to the $\mathrm{C}$ group and increased in the MD group compared to the $\mathrm{M}$ group at week 4 (C vs. $\mathrm{M}$ vs. $\mathrm{MD} ; 100.0 \%$ vs. $66.7 \%$ vs. $73.3 \%$ ).

\section{ET-1 and ERA Expressions Were Reduced After Modafinil Administration}

We investigated ET-1 and endothelin receptor A (ERA) expression levels in lung tissues by western blot analysis and immunohistochemistry (Figure 4). First, in western blot analysis, the protein expression levels of ET-1 was significantly

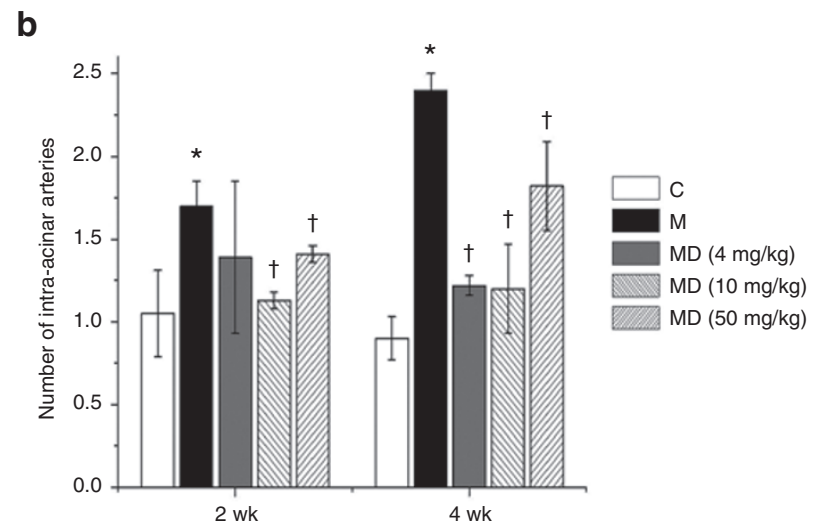

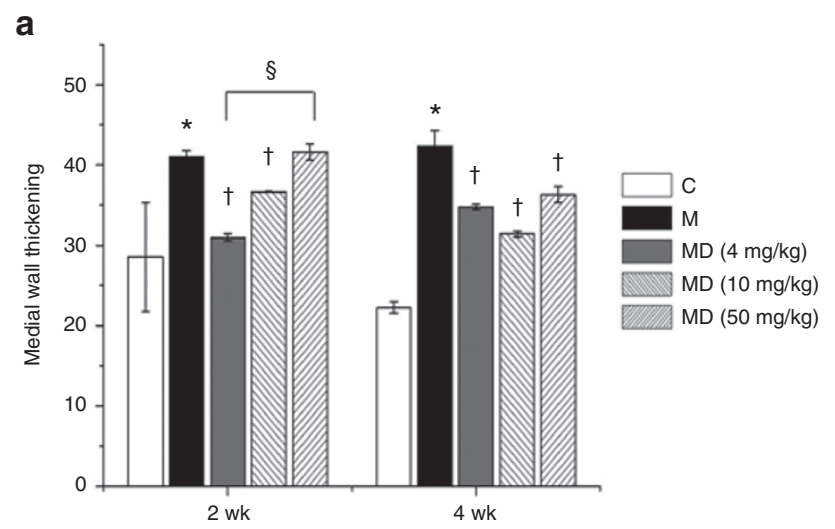
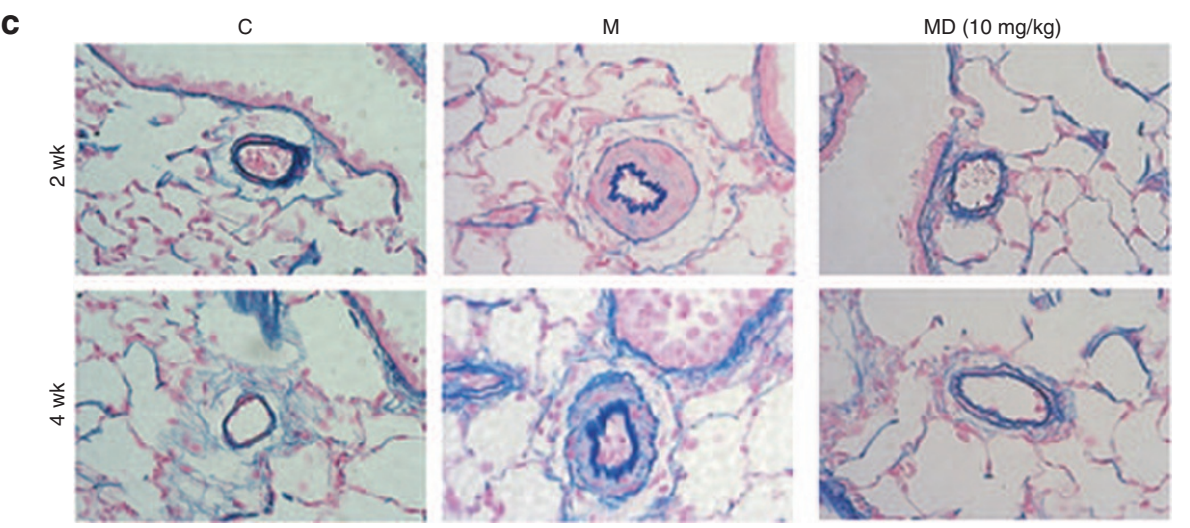

Figure 3. Changes of pulmonary pathology after modafinil administration in MCT-induced PAH rat. (a) The medial layer of the pulmonary arterioles thickened progressively after the MCT injection. The medial wall thickenesses were significantly reduced in the MD groups at weeks 2 and 4 . (b) The number of intraacinar arteries were decreased in the MD groups at weeks 2 and 4 (c) Representative images of pulmonary arteriole in each group ( $\times 400)$. C, control group; $M$, monocrotaline group; $M D$, modafinil group. ${ }^{*} P<0.05 \mathrm{C}$ vs. $M,{ }^{\dagger} P<0.05 \mathrm{M}$ vs. $\mathrm{MD},{ }^{\S} P<0.05$ among the $\mathrm{MD}$ groups. 

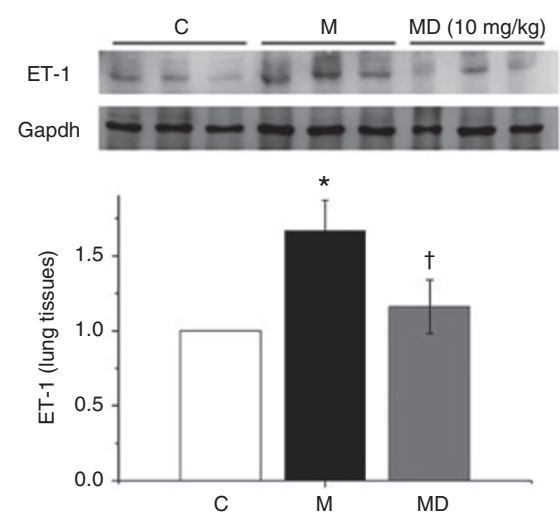

c

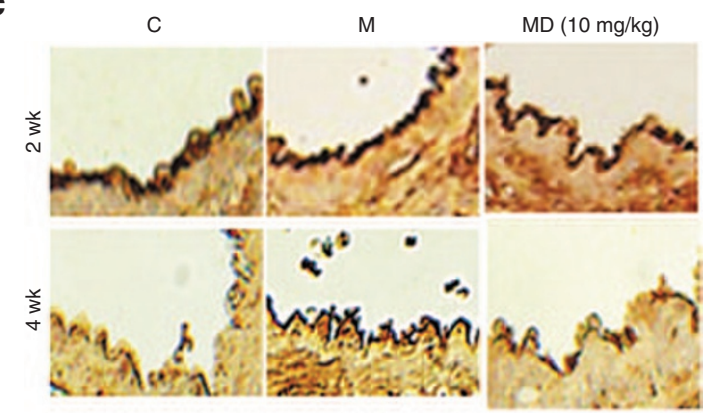

b
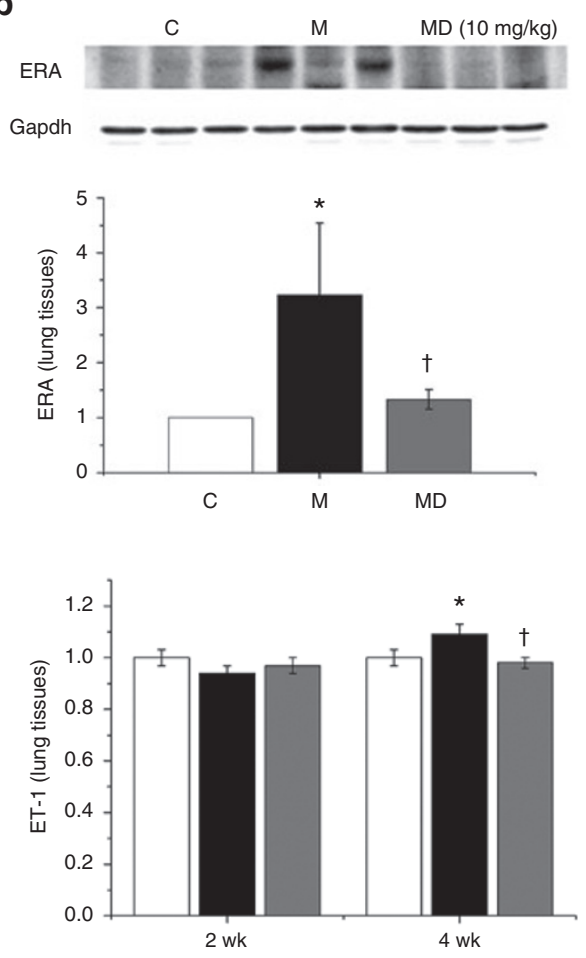

d

C

M

MD (10 mg/kg)

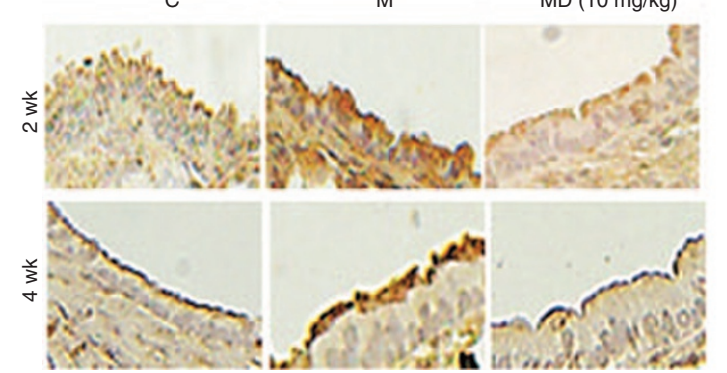

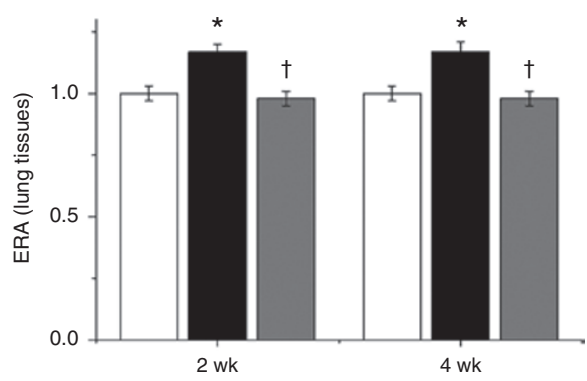

e
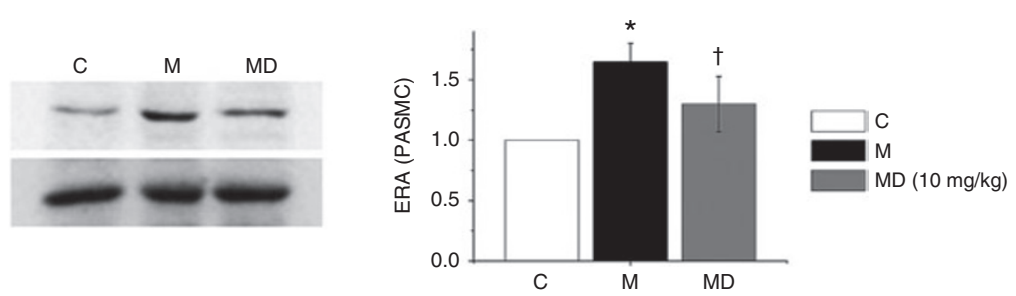

Figure 4. Downregulation of ET-1 and ERA expression after modafinil treatment. (a-d) ET-1 expression was decreased after modafinil administration in lung tissues. (e) Modafinil significantly decreased MCT-induced ERA expression in PASMCs. C, control group; ERA, endothelin receptor A; ET-1, endothelin-1; $M$, monocrotaline group; $M D$, modafinil group. ${ }^{*} P<0.05 C$ vs. $M,{ }^{+} P<0.05, M$ vs. MD.

increased in the $\mathrm{M}$ group compared to the $\mathrm{C}$ group and significantly decreased in the MD group compared to the M group at week 4 in the lung tissues. The protein expression levels of ERA were significantly increased in the M group compared to the $\mathrm{C}$ group and significantly decreased in the MD group compared to the $\mathrm{M}$ group at week 4. Second, in immunohistochemistry, ET-1 expression was significantly increased in the $\mathrm{M}$ group compared to the $\mathrm{C}$ group and significantly decreased in the MD group compared to the M group at week 4. ERA expression was significantly increased in the $\mathrm{M}$ group at weeks 2 and 4 and significantly decreased in the MD group at weeks 2 and 4. Third, in PASMCs, the expression level of ERA was significantly increased in the $M$ group and significantly decreased after modafinil (100 nmol/l) treatment. Because of this, we considered that modafinil contributed to the improvement of PAH by controlling expression levels of ET- 1 and ERA.

\section{KCa3.1 Channel Expression Was Reduced After Modafinil Administration}

We investigated changes of KCa3.1 channel expression levels after modafinil administration by western blot analysis and immunohistochemistry in lung tissues. First, KCa3.1 channel 
a

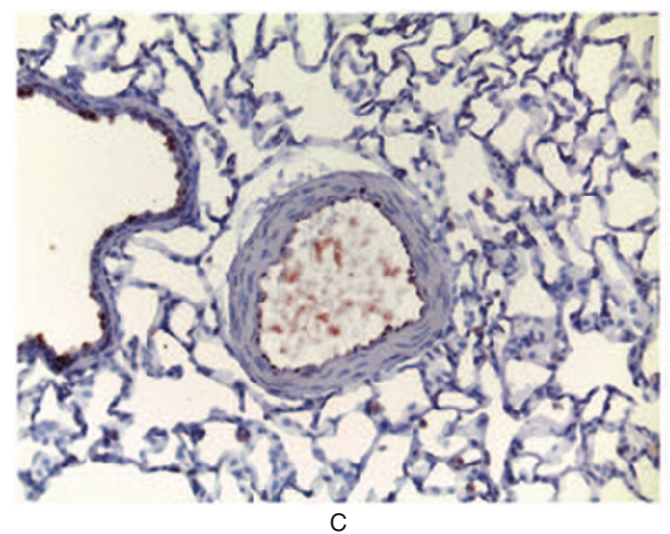

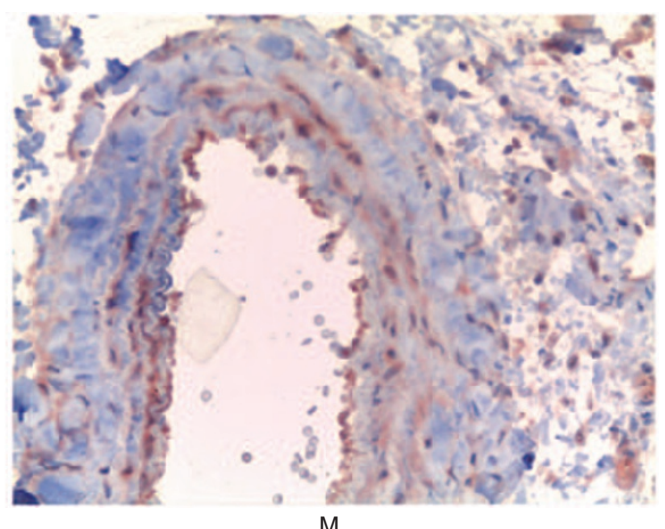

b

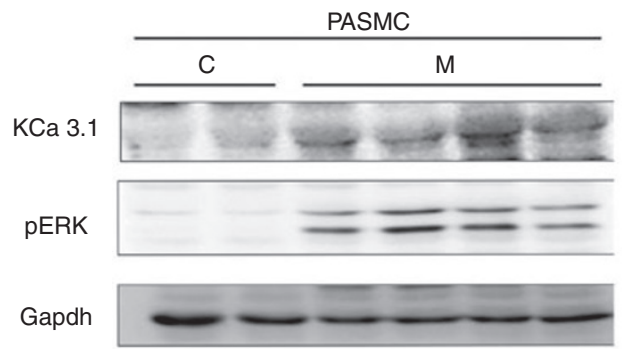

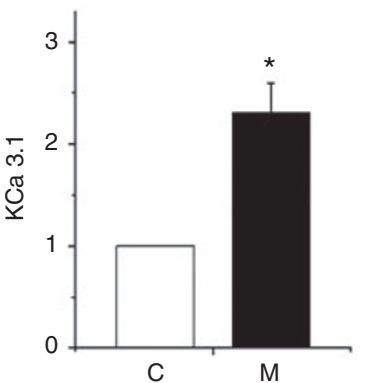

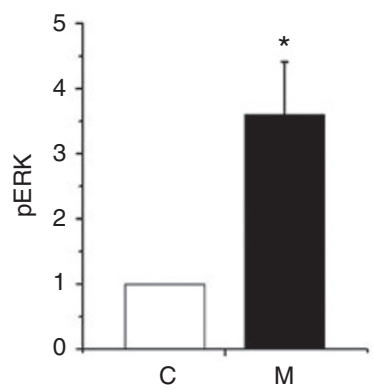

Figure 5. Upregulation of KCa3.1 expression in PASMCs of MCT-induced PAH rat. (a) KCa3.1 channel expression was increased in thickened medial layers of pulmonary arteriole in M group comprared with C group $(\times 400)$. (b) KCa3.1 channel and pERK expression were increased in PASMCs of M group compared with C group. C, control group; $M$, monocrotaline group; ${ }^{*} P<0.05 \mathrm{C}$ vs. $\mathrm{M}$.

a

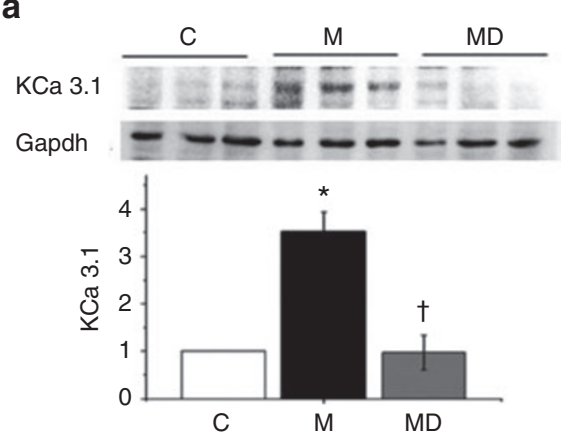

C

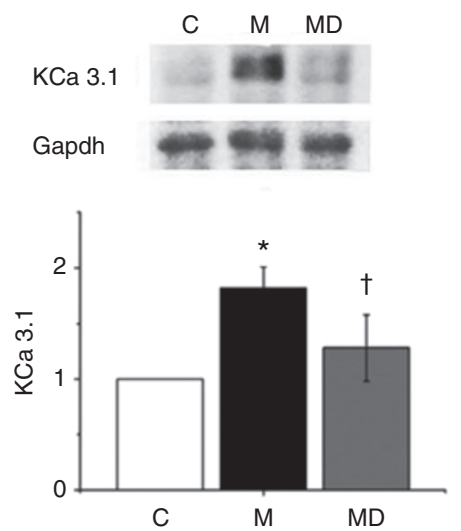

b
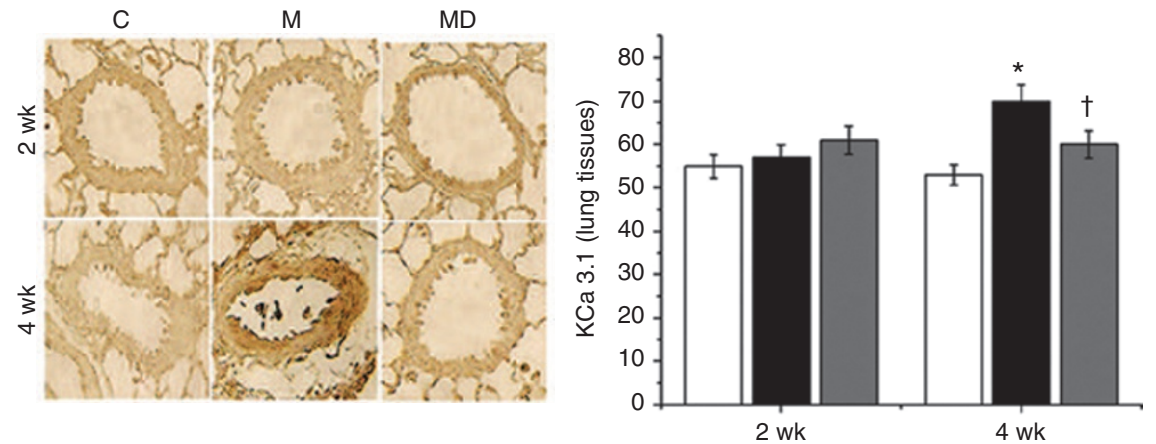

d

e
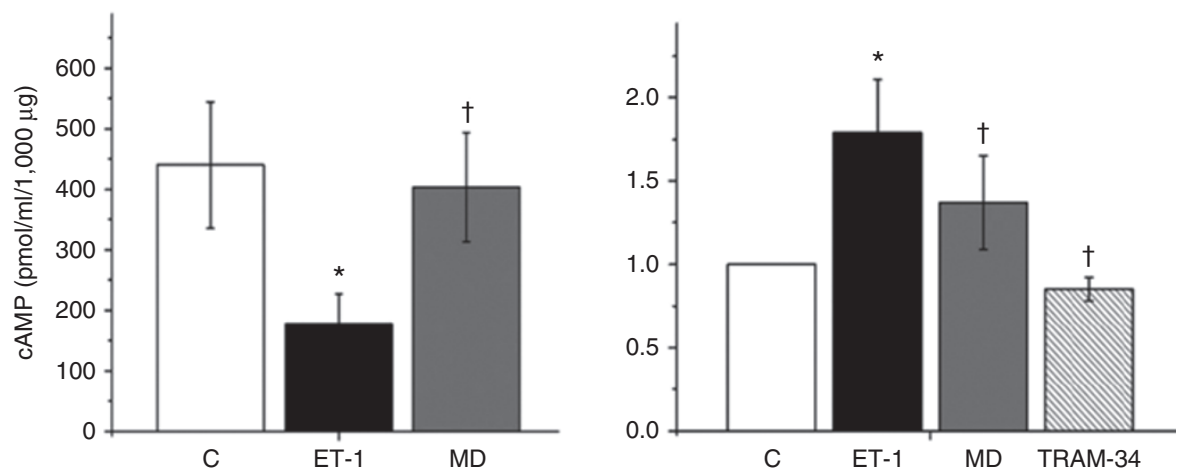

Figure 6. Downregulation of KCa3.1 expression in the lung tissues after modafinil treatment. (a,b) KCa3.1 expression was decreased in the lung tissues after modafinil adnimistration. (c) MD decreased MCT-induced KCa3.1 expression in PASMCs. (d) MD increased intracellular cyclic adenosine monophosphate (CAMP) accumulation. (e) MD and TRAM-34 suppressed ET-1-induced PASMC proliferation. C, control group; M, monocrotaline group; MD, modafinil group; ${ }^{P} P<0.05$ C vs. ET-1 or C vs. ET-1, ${ }^{+} P<0.05 \mathrm{M}$ vs. MD or ET-1 vs. MD or ET-1 vs. TRAM-34. 


\section{Articles | Lee etal.}

expression was increased in the medial walls of the pulmonary arterioles in the $\mathrm{M}$ group compared with the $\mathrm{C}$ group. KCa3.1 channel expression levels of primary PASMCs of the $M$ group was significantly increased compared to the $C$ group (Figure 5). Second, in western blot analysis and immunohistochemistry, KCa3.1 channel expression levels were significantly increased in the $\mathrm{M}$ group compared to the $\mathrm{C}$ group and significantly decreased in the MD group compared to the $M$ group at week 4. Third, in PASMCs of MCT-induced PAH rats, KCa3.1 expression was significantly decreased after modafinil (100 nmol/l) treatment. Fourth, intracellular cAMP level was significantly decreased by ET-1 in PASMCs. Modafinil and forskolin significantly increased the cAMP levels. Forskolin is commonly used to raise intracellular cAMP level. Fifth, ET-1induced proliferation was significantly decreased by modafinil and KCa3.1 channel-specific blocker, TRAM-34 (Figure 6). Therefore, we concluded that modafinil decreased PASMC proliferation by $\mathrm{KCa} 3.1$ dependent pathway.

\section{Protein Expressions of Bax, VEGF, TNF- $\alpha$, and IL- 6 Were Decreased in Lung Tissues After Modafinil Administration} We investigated other protein expressions such as Bcl-2associated X (Bax), vascular endothelial growth factor (VEGF), tumor necrosis factor- $\alpha$ (TNF- $\alpha$ ), interleukin-6 (IL-6) in the lung tissues (Figure 7). First, Bax expression (apoptosis marker) was significantly decreased after modafinil administration at week 4. Second, VEGF (angiogenesis marker) was significantly decreased after modafinil treatment at week4. Third, TNF- $\alpha$ and IL- 6 (inflammation marker) were also decreased after modafinil administration at weeks 2 and 4 . We confirmed reduced expression levels of Bax, VEGF, TNF- $\alpha$, and IL- 6 by immunohistochemistry. Therefore, the data suggests that modafinil decreased Bax, VEGF, TNF- $\alpha$, and IL-6 expression level in PASMCs.

\section{DISCUSSION}

Our main conclusion is that modafinil did improve PAH in our rat model by reducing RVP and medial wall thickening of pulmonary arterioles.

The main purpose of this study was to determine the effects of modafinil on PAH rat model. We have the following important results: (i) modafinil did reduce RVP and improve survival rate in PAH rats; (ii) it had an effect on the improvement of $\mathrm{RV}$ hypertrophy which is an indicator of RV pressure overload caused by elevated pulmonary artery pressure; (iii) it decreased the number of intra-acinar arteries and medial wall thickening in pulmonary arterioles which are an index of vascular remodeling.

We have two hypotheses for the mechanisms of how modafinil decreases medial wall thickening: (i) modafinil has a vasodilatory and antiproliferative effects through a decrease in expression of ET-1 and ERA in the lung tissues. The reason why we think ET-1 is involved is as follows. There have been reports that other cAMP elevating drugs similar to modafinil decrease ET-1 release and ERA protein expression level in PASMCs. In Wort et al. (23) study, there was a report that cicaprost inhibited endogenous endothlin-1 release from human PASMCs. In Davie et al. (24) study, cicaprost inhibited ET-1 release from PASMCs, increased cAMP levels and suppressed DNA synthesis; (ii) modafinil shows an antiproliferative effect by the reduction of KCa3.1 channel expression in PASMCs in the lung tissues. This hypothesis was based on a previous report that KCa3.1 channel is involved in airway smooth muscle (22).

We think that modafinil's effects on decrease in pulmonary vessel contraction and remodeling secondarily leads to an improvement in apoptosis, neovascularization and inflammation (Bax, VEGF, IL-6, and TNF- $\alpha$ gene expression) in $\mathrm{PAH}$ rats. These findings do support our hypothesis that oral modafinil administration improves PAH.

The positive effect of modafinil on PAH might be due to the involvement of dual pathways. The first is ET-1-dependent pathway. In this study, modafinil decreased ET-1 and ERA expression in the lung tissues. ET-1 is a well-known vasoconstrictor and is increased in PAH patients (25). Modafinil is a cAMP-elevating agent similar with iloprost currently being using for PAH (20). According to Wood et al. ET-1 expression was decreased by elevated cAMP levels (23). There was a report that cAMP induces upregulation of ERA mRNA level. It suggests that modafinil decreases ET-1 by elevating CAMP levels $(23,26)$. However, the mechanisms that ET-1 elevates cAMP levels are not clear.

The second is the KCa3.1-dependent pathway. In our study, KCa3.1 channel expression significantly reduced in the lung tissues, especially PASMCs, by the effect of modafinil. KCa3.1 channels are phosphorylated by cAMP-dependent pathway (20). Cruse et al. (21) reported that the blockade of KCa3.1 channels attenuated human lung mast cell proliferation and migration. According to Shepherd et al. (22) KCa3.1 channels regulated smooth muscle proliferation of the human lung airway. In Strøbæk et al. (27) study, KCa3.1 channels attenuated human lung mast cell blocker NS6180. In our study, modafinil showed the ability to decrease in KCa3.1 channels expression at a very low concentration at $100 \mathrm{nmol} / \mathrm{l}$ in vitro. So, our conclusion is that decreased KCa3.1 expression is involved in reduced PASMCs proliferation in PAH. Decreased RVP is because of a reduction in PASMCs proliferation, which enlarges the inner space of the pulmonary vessels.

We think that modafinil improves PAH by reducing excessive vascular SMC contraction via ET-1 and proliferation via KCa3.1 channel. Decrease in vasoconstriction and PASMCs proliferation also leads to a decrease in apoptosis markers (Bax), angiogenesis marker (VEGF), and inflammatory markers (TNF- $\alpha$, IL-6).

Modafinil has a similar effect on PAH rats as other current therapies, such as ETR antagonists, phosphodiesterease type 5 inhibitor, and prostacyclin (1). Even though these treatments have not led to a cure yet, these treatments have increased the survival rate and quality of life. From our previous study of the same PAH models, RVP and the number of intra-acinar arteries were decreased at weeks 2 and 4 after bosentan $(25 \mathrm{mg} / \mathrm{kg} / \mathrm{d})$ administrations. Medial wall thickening was also decreased at week 4 after bosentan treatment $(28,29)$. 
a

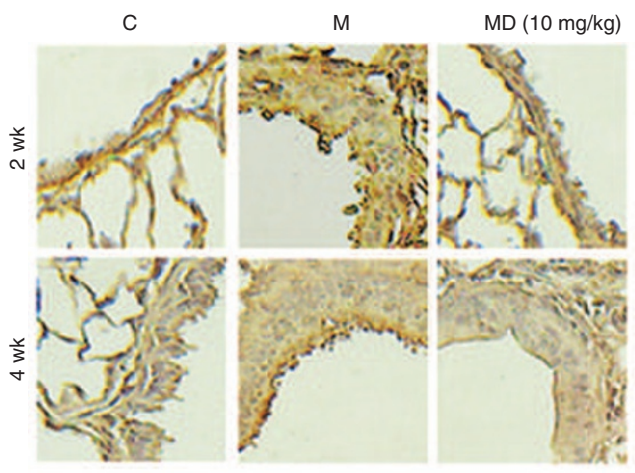

b

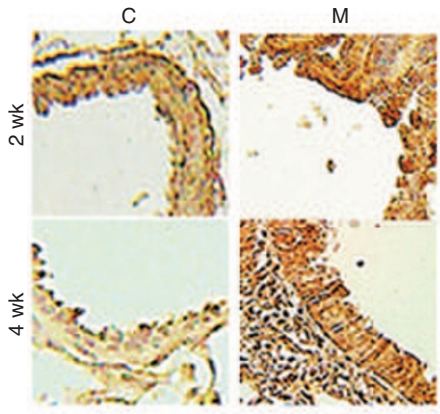

$\mathrm{MD}(10 \mathrm{mg} / \mathrm{kg})$
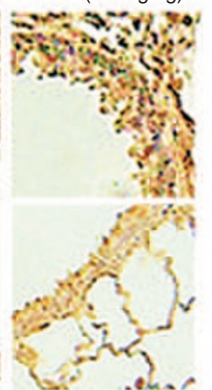

C

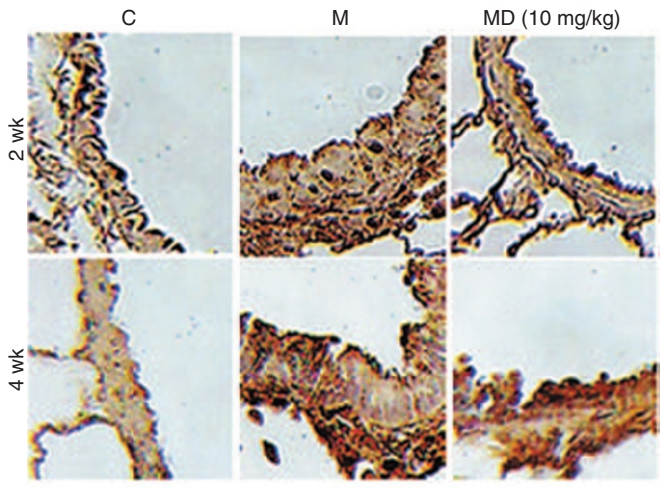

d

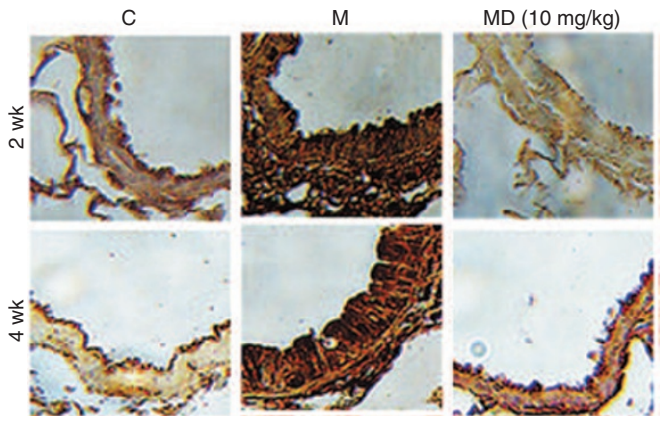

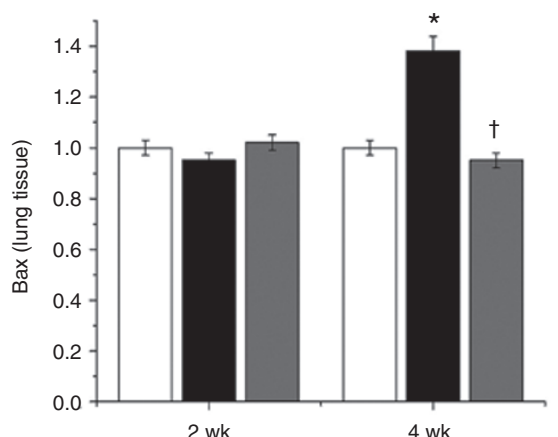
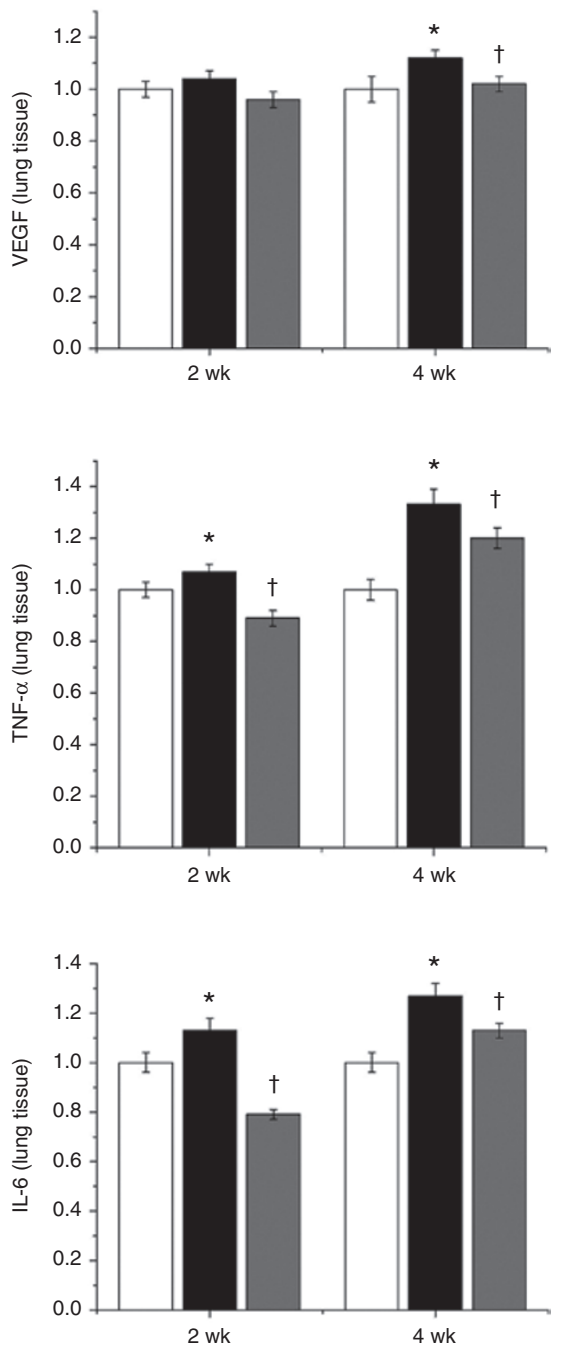

Figure 7. Changes of Bax, VEGF, TNF- $\alpha$, and IL- 6 in the lung tissues of PAH rat. Bax, VEGF, TNF- $\alpha$, and IL-6 expressions were decreased after modafinil administration in the lung tissues. Bax, Bcl-2-associated X protein; $C$, control group; IL-6, interleukin-6; M, monocrotaline group; MD, modafinil group; TNF- $\alpha$, tumor necrosis factor- $\alpha$; VEGF, vascular endothelial growth factor; ${ }^{*} P<0.05 C$ vs. $M,{ }^{\dagger} P<0.05$ M vs. MD.

Our previous infliximab (TNF- $\alpha$ antagonist) study $(5 \mathrm{mg} / \mathrm{kg}$, single sc injection) on the MCT-induced PAH model was as follows: medial wall thickening was decreased at week 1 , the number of intra-acinar arteries at weeks 2 and 4 but there was no effect on RVP (30). Clozel et al. (31) used an injection of the same dose of MCT $(60 \mathrm{mg} / \mathrm{kg})$ as our study. Their results were decreased RV/body weight at week 4 after treatment of bosen$\tan (300 \mathrm{mg} / \mathrm{kg} / \mathrm{d})$ and $/$ or sildenafil $(100 \mathrm{mg} / \mathrm{kg} / \mathrm{d})$. Modafinil's inhibitory effect on RV hypertrophy was similar with bosentan or sildenafil.

However, it was difficult to compare the data itself directly because their sacrifice time and MCT dose was different from 
ours. Therefore, the effects of modafinil were as good as current therapies compared with previous studies.

Modafinil has several advantages as a new therapeutic approach under development for PAH compared with other current medication.

First, it has few safety problem and less side effects. Modafinil treatment has been associated with minimal toxicity and low propensity for abuse (32). The second advantage is both relaxation and antiproliferative effect in vascular smooth muscle simultaneously. In our study, modafinil has effects on decreasing KCa3.1 expression in the lung tissues of MCT-induced PAH rats. KCa3.1 blocker TRAM-34 can possibly reduce proliferation but it cannot induce relaxation (33). The third is that it can be delivered orally (34). The mechanisms of modafinil are similar with prostacyclin, elevating cAMP. Prostacyclin is administrated by the intravenous, inhaled, and subcutaneous routes but not by an oral route (1). Fourth, it has a long halflife. Prostacyclin has a short half-life compared to modafinil which is half-life is $15 \mathrm{~h}$. So, prostacyclin is inconvenient to take. Fifth, it is relatively low in price.

We also investigated the optimal dose of MD. We think that it might be as $10 \mathrm{mg} / \mathrm{kg}$. There were no differences in the effects according to modafinil dosage in PAH. RVP and RV/ $\mathrm{LV}+\mathrm{S}$ ratio were not significantly different according to dose. However, there was no effect on medial wall thickening in MD $50 \mathrm{mg} / \mathrm{kg}$ at week 2 and the number of intra-acinar arteries in $\mathrm{MD} 4 \mathrm{mg} / \mathrm{kg}$ at week 2. Therefore, we mainly used $10 \mathrm{mg} /$ $\mathrm{kg}$ modafinil in this study. Currently, $200 \mathrm{mg} / \mathrm{d}$ of modafinil is used in narcolepsy, sleep apnea/hypopnea syndrome, and shift fork sleep disorder according to the Provigil prescription information (32).

Modafinil may be used in treating cardiovascular disease and has potential to induce vessel relaxation and inhibit excessive proliferation of vascular cells.

In conclusion, modafinil has an effect on the suppression of PAH. Furthermore, this study suggests that modafinil might be a new medicine for PAH. Further studies are needed to determine what pathways are involved in improvement of $\mathrm{PAH}$ by modafinil.

\section{METHODS}

\section{Animal Model}

Six-week-old male Sprague-Dawley rats were obtained from Orient Bio (Orient Bio, Seongnam, Korea). The rats were kept at a constant room temperature with a natural day-night cycle. PAH was induced in rats by MCT (Sigma Chemicals, St. Louis, MO) injection subcutaneously. The rats were grouped as follows: the control (C) group $(n=$ $30)$; the M group $(n=64$, MCT $60 \mathrm{mg} / \mathrm{kg})(29,30)$; the MD group $(n=70$, MCT $60 \mathrm{mg} / \mathrm{kg}+$ modafinil). The MD group was subdivided to three subgroups to determine therapeutic optimal dose of modafinil $(4,10$, and $50 \mathrm{mg} / \mathrm{kg})$. The study protocols were approved by the Institutional Review Board of the School of Medicine of Ewha Womans University. All studies were approved by Institutional Animal Card and Use Committee (IACUC No. 13-0238).

\section{Hemodynamics}

Rats were anesthetized intraperitonealy with Zoletil (Virbac, Carros, France) and Rompun (Bayer Korea, Seoul, Korea). Hemodynamic parameters were recorded at baseline, weeks 2 and 4 . The catheter was placed in the RV to estimate the mean RVP.

\section{Pathology in the Lung Tissues}

Medial wall thickening and the number of intra-acinar arteries assessed as previously described (35).

\section{Masson's Trichrome Staining in RV Tissues}

Masson's trichrome staining was performed. It is used in order to observe the degree of collagen fiber penetrating using light microscopy. The photographs were processed through an image analysis program (anaylySIS FIVE, Olympus, Tokyo, Japan) and the degree of collagen fiber was measured by image J (NIH imageJ software version $1.43 \mathrm{u}$ ). To verify the differences within each group, we implemented Duncan's multiple range test.

\section{Western Blot Analysis in the Lung and RV Tissues}

Whole tissue extracts from rat lung were prepared by homogenization in lysis buffer (Proprep, Intron, Seongnam, Korea). Lysates were normalized and separated on $8-12 \%$ polyacrylamide gels and transferred to nitrocellulose blotting membranes (GE Healthcare: formerly Amersham Bioscience, Munich, Germany). After staining with Ponceau red, membrane blocked with $5 \%$ bovine serum albumin $1 \mathrm{~h}$ at room temperature followed by incubation with primary antibodies; ET-1 (Abcam, Cambridge, UK), KCa3.1 (Sigma-Aldrich), ERA, Bax, Bcl-2, VEGF, TNF- $\alpha$, and IL-6 (Santacruz Biotechnology, Santa Cruz, CA) overnight. Nitrocellulose membranes were then washed in TBS$\mathrm{T}$. The membranes were incubated with horseradish conjugated secondary antibodies for $1 \mathrm{~h}$ at room temperature. The bound secondary antibody was detected by ECL western blotting detection reagents (GE Healthcare: formerly Amersham Bioscience, Buckinghamshire, UK).

\section{Immunohistochemical Analysis in Lung Tissues}

Immunohistochemical analysis was performed with antibodies to ET-1, KCa3.1, ERA, Bax, Bcl-2, VEGF, TNF- $\alpha$, and IL-6. Paraffin blocks that were routinely processed after $10 \%$ neutral buffered formalin (Sigma-Aldrich, St. Louis, MO) fixation in the lung and heart tissues. Heat antigen retrieval was achieved by boiling of tissue sections in antigen retrieval solution $\mathrm{pH} 6.0$ or $\mathrm{pH} 9.0$ (Dako, Carpinteria, CA) for $10 \mathrm{~min}$ in microwave prior to incubation at $4{ }^{\circ} \mathrm{C}$ overnight with primary antibodies. After incubation with the appropriate biotinylated secondary antibodies for $30 \mathrm{~min}$ at $4{ }^{\circ} \mathrm{C}$ and subsequently with a streptavidin (Dako, Kyoto, Japan), color development was done using 3-amino-9-ethylcarbazole (AEC, Kyoto, Japan). The photographs were processed through an image analysis program (analySIS FIVE, Olympus, Tokyo, Japan) and the degree of staining densities was measured by image J.

\section{PASMC Isolation}

Normal Sprague-Dawley rats or MCT-induced PAH rats were anesthetized and the lungs and heart were excised. The pulmonary arteries were dissected at $4{ }^{\circ} \mathrm{C}$ filtered phosphate-buffered saline. Arteries were cleaned of connective tissue, cut into small pieces, and placed in a tube containing enzyme solution. PASMCs were enzymatically isolated from pulmonary arteries at $37^{\circ} \mathrm{C}$. The pulmonary arterial tissues were flushed a few times. After digestion, the resulting dispersed PASMCs were centrifuged 1,200 rpm, $5 \mathrm{~min}$ and replaced to new media. Only cells with normal morphology were studied.

\section{cAMP Production}

Intracellular cAMP level was measured after stimulation by ET-1 or ET-1 + MD (100 nmol/l) at $24 \mathrm{~h}$. Intracellular cAMP accumulation level was assessed using cAMP parameter assay (R\&D systems, Minneapolis, $\mathrm{MN}$ ) as described.

\section{Proliferation Assay}

Proliferation assay was processed after stimulation by ET-1 or ET-1 + MD or ET-1 + TRAM-34 at 24h. Proliferation was assessed using EZ-Cytox Cell viability assay kit was (Daeil Lab Service, Seoul, Korea).

\section{Statistical Analysis}

Results are expressed as the mean \pm SD. Data were analyzed using SPSS (SPSS v22.0, Chicago, IL) for windows. The Mann-Whitney test was used to test for significant differences between the $C$ and $M$ group. The Kruskal-Wallis test was used to test for significant differences between M group and MD group. If significant, we conducted Pairwise by Conover method among the MD groups. 


\section{STATEMENT OF FINANCIAL SUPPORT}

This research was supported by Basic Science Research Program through the National Research Foundation of Korea funded by the Ministry of Education (2013R1A1A3004619).

Disclosure: Neither of the authors has any conflict of interest to disclose.

\section{REFERENCES}

1. Montani D, Günther S, Dorfmüller P, et al. Pulmonary arterial hypertension. Orphanet J Rare Dis 2013;8:97.

2. Rabinovitch M. Molecular pathogenesis of pulmonary arterial hypertension. J Clin Invest 2008;118:2372-9.

3. Voelkel NF, Gomez-Arroyo J, Abbate A, Bogaard HJ, Nicolls MR. Pathobiology of pulmonary arterial hypertension and right ventricular failure. Eur Respir J 2012;40:1555-65.

4. Eddahibi S, Humbert M, Fadel E, et al. Serotonin transporter overexpression is responsible for pulmonary artery smooth muscle hyperplasia in primary pulmonary hypertension. J Clin Invest 2001;108:1141-50.

5. Dias-Neto M, Luísa-Neves A, Pinho S, et al. Pathophysiology of infantile pulmonary arterial hypertension induced by monocrotaline. Pediatr Cardiol 2015;36:1000-13.

6. Zhao LM, Su XL, Wang Y, Li GR, Deng XL. KCa3.1 channels mediate the increase of cell migration and proliferation by advanced glycation endproducts in cultured rat vascular smooth muscle cells. Lab Invest 2013;93:159-67.

7. Su XL, Zhang H, Yu W, Wang S, Zhu WJ. Role of KCa3.1 channels in proliferation and migration of vascular smooth muscle cells by diabetic rat serum. Chin J Physiol 2013;56:155-62.

8. Toyama K, Wulff H, Chandy KG, et al. The intermediate-conductance calcium-activated potassium channel KCa3.1 contributes to atherogenesis in mice and humans. J Clin Invest 2008;118:3025-37.

9. Gomez-Arroyo JG, Farkas L, Alhussaini AA, et al. The monocrotaline model of pulmonary hypertension in perspective. Am J Physiol Lung Cell Mol Physiol 2012;302:L363-9.

10. Stenmark KR, Fagan KA, Frid MG. Hypoxia-induced pulmonary vascular remodeling: cellular and molecular mechanisms. Circ Res 2006;99:675-91.

11. Hessel MH, Steendijk P, den Adel B, Schutte CI, van der Laarse A. Characterization of right ventricular function after monocrotaline-induced pulmonary hypertension in the intact rat. Am J Physiol Heart Circ Physiol 2006;291:H2424-30.

12. Stenmark KR, Meyrick B, Galie N, Mooi WJ, McMurtry IF. Animal models of pulmonary arterial hypertension: the hope for etiological discovery and pharmacological cure. Am J Physiol Lung Cell Mol Physiol 2009;297:1013-32.

13. Wilkins MR, Ali O, Bradlow W, et al.; Simvastatin Pulmonary Hypertension Trial (SiPHT) Study Group. Simvastatin as a treatment for pulmonary hypertension trial. Am J Respir Crit Care Med 2010;181:1106-13.

14. Haddad F, Kudelko K, Mercier O, Vrtovec B, Zamanian RT, de Jesus Perez V. Pulmonary hypertension associated with left heart disease: characteristics, emerging concepts, and treatment strategies. Prog Cardiovasc Dis 2011;54:154-67.

15. Galiè N, Ghofrani HA, Torbicki A, et al.; Sildenafil Use in Pulmonary Arterial Hypertension (SUPER) Study Group. Sildenafil citrate therapy for pulmonary arterial hypertension. N Engl J Med 2005;353:2148-57.

16. Hoeper MM, Schwarze M, Ehlerding S, et al. Long-term treatment of primary pulmonary hypertension with aerosolized iloprost, a prostacyclin analogue. N Engl J Med 2000;342:1866-70.

17. Benza RL, Miller DP, Barst RJ, Badesch DB, Frost AE, McGoon MD. An evaluation of long-term survival from time of diagnosis in pulmonary arterial hypertension from the REVEAL Registry. Chest 2012;142:448-56.
18. Mignot EJ. A practical guide to the therapy of narcolepsy and hypersomnia syndromes. Neurotherapeutics 2012;9:739-52.

19. Ballon JS, Feifel D. A systematic review of modafinil: Potential clinical uses and mechanisms of action. J Clin Psychiatry 2006;67:554-66.

20. Choi S, Kim MY, Joo KY, et al. Modafinil inhibits K(Ca)3.1 currents and muscle contraction via a cAMP-dependent mechanism. Pharmacol Res 2012;66:51-9.

21. Cruse G, Duffy SM, Brightling CE, Bradding P. Functional KCa3.1 $\mathrm{K}+$ channels are required for human lung mast cell migration. Thorax 2006;61:880-5.

22. Shepherd MC, Duffy SM, Harris T, et al. KCa3.1 Ca2+ activated K+ channels regulate human airway smooth muscle proliferation. Am J Respir Cell Mol Biol 2007;37:525-31.

23. Wort SJ, Mitchell JA, Woods M, Evans TW, Warner TD. The prostacyclin-mimetic cicaprost inhibits endogenous endothelin-1 release from human pulmonary artery smooth muscle cells. J Cardiovasc Pharmacol 2000;36:S410-3.

24. Davie N, Haleen SJ, Upton PD, et al. ET(A) and ET(B) receptors modulate the proliferation of human pulmonary artery smooth muscle cells. Am J Respir Crit Care Med 2002;165:398-405.

25. Rubens C, Ewert R, Halank M, et al. Big endothelin-1 and endothelin-1 plasma levels are correlated with the severity of primary pulmonary hypertension. Chest 2001;120:1562-9.

26. Nishimura J, Chen X, Jahan H, Shikasho T, Kobayashi S, Kanaide H. cAMP induces up-regulation of ETA receptor mRNA and increases responsiveness to endothelin-1 of rat aortic smooth muscle cells in primary culture. Biochem Biophys Res Commun 1992;188:719-26.

27. Strøbæk D, Brown DT, Jenkins DP, et al. NS6180, a new K(Ca) 3.1 channel inhibitor prevents T-cell activation and inflammation in a rat model of inflammatory bowel disease. Br J Pharmacol 2013;168:432-44.

28. Lim KA, Kim KC, Cho MS, Lee BE, Kim HS, Hong YM. Gene expression of endothelin-1 and endothelin receptor a on monocrotaline-induced pulmonary hypertension in rats after bosentan treatment. Korean Circ J 2010;40:459-64.

29. Koo HS, Kim KC, Hong YM. Gene expressions of nitric oxide synthase and matrix metalloproteinase-2 in monocrotaline-induced pulmonary hypertension in rats after bosentan treatment. Korean Circ J 2011;41:83-90.

30. Kwon JH, Kim KC, Cho MS, Kim HS, Sohn S, Hong YM. An inhibitory effect of tumor necrosis factor-alpha antagonist to gene expression in monocrotaline-induced pulmonary hypertensive rats model. Korean J Pediatr 2013;56:116-24.

31. Clozel M, Hess P, Rey M, Iglarz M, Binkert C, Qiu C. Bosentan, sildenafil, and their combination in the monocrotaline model of pulmonary hypertension in rats. Exp Biol Med (Maywood) 2006;231:967-73.

32. Kumar R. Approved and investigational uses of modafinil: an evidencebased review. Drugs 2008;68:1803-39.

33. Grgic I, Eichler I, Heinau P, et al. Selective blockade of the intermediateconductance $\mathrm{Ca} 2+$-activated $\mathrm{K}+$ channel suppresses proliferation of microvascular and macrovascular endothelial cells and angiogenesis in vivo. Arterioscler Thromb Vasc Biol 2005;25:704-9.

34. Parnell H, Quirke G, Farmer S, Adeyemo S, Varney V. The successful treatment of hypercapnic respiratory failure with oral modafinil. Int J Chron Obstruct Pulmon Dis 2014;9:413-9.

35. Kim KC, Lee JC, Lee H, Cho MS, Choi SJ, Hong YM. Changes in caspase-3, B cell leukemia/lymphoma-2, interleukin-6, tumor necrosis factor- $\alpha$ and vascular endothelial growth factor gene expression after human umbilical cord blood derived mesenchymal stem cells transfusion in pulmonary hypertension rat models. Korean Circ J 2016;46:79-92. 